University of Wollongong

Research Online

Faculty of Engineering and Information

Faculty of Engineering and Information

Sciences - Papers: Part A

Sciences

$1-1-2014$

Highly efficient polarization-independent grating coupler used in silicabased hybrid photodetector integration

Hongqiang Li

Tianjin Polytechnic University

Yu Liu

Tianjin Polytechnic University, yl751@uowmail.edu.au

Meiling Zhang

Tianjin Polytechnic University

Wenqian Zhou

Tianjin Polytechnic University

Cheng Zhang

Tianjin Polytechnic University

See next page for additional authors

Follow this and additional works at: https://ro.uow.edu.au/eispapers

Part of the Engineering Commons, and the Science and Technology Studies Commons

Research Online is the open access institutional repository for the University of Wollongong. For further information contact the UOW Library: research-pubs@uow.edu.au 


\title{
Highly efficient polarization-independent grating coupler used in silica-based hybrid photodetector integration
}

\author{
Abstract \\ A highly efficient polarization-independent output grating coupler was optimized and designed based on \\ silicon-on-insulator used for silica-based hybrid photodetector integration in an arrayed waveguide grating \\ demodulation-integrated microsystem. The finite-difference time-domain (FDTD) method optimizes \\ coupling efficiency by enabling the design of the grating period, duty cycle, etch depth, grating length, and \\ polarization-dependent loss (PDL). The output coupling efficiencies of both the transverse electric (TE) \\ and transverse magnetic (TM) modes are higher than $60 \%$ at 1517 to $1605 \mathrm{~nm}$ and similar to $67 \%$ at \\ around $1550 \mathrm{~nm}$. The designed grating exhibits the desired property at the 3-dB bandwidth of $200 \mathrm{~nm}$ \\ from 1450 to $1650 \mathrm{~nm}$ and a PDL <0.5 dB of $110 \mathrm{~nm}$ from 1513 to $1623 \mathrm{~nm}$. The power absorption \\ efficiency at $1550 \mathrm{~nm}$ for TE and TM modes reaches $78 \%$ and $70 \%$, respectively. Both the power \\ absorption efficiency of TE mode and that of TM mode are over $70 \%$ in a broad band of 1491 to $1550 \mathrm{~nm}$. \\ Disciplines \\ Engineering | Science and Technology Studies

\section{Publication Details} \\ Li, H., Liu, Y., Zhang, M., Zhou, W., Zhang, C., Li, E., Miao, C. \& Tang, C. (2014). Highly efficient polarization- \\ independent grating coupler used in silica-based hybrid photodetector integration. Optical Engineering, 53 \\ (5), 057105-1-057105-6.
}

\section{Authors}

Hongqiang Li, Yu Liu, Meiling Zhang, Wenqian Zhou, Cheng Zhang, Enbang Li, Changyun Miao, and Chunxiao Tang 


\section{Optical Engineering}

\section{Highly efficient polarization- independent grating coupler used in silica-based hybrid photodetector integration}

Hongqiang Li

Yu Liu

Meiling Zhang Wenqian Zhou

Cheng Zhang

Enbang Li

Changyun Miao

Chunxiao Tang 


\title{
Highly efficient polarization-independent grating coupler used in silica-based hybrid photodetector integration
}

\author{
Hongqiang Li, ${ }^{a, *}$ Yu Liu, ${ }^{a}$ Meiling Zhang,, Wenqian Zhou, ${ }^{a}$ Cheng Zhang, ${ }^{a}$ Enbang Li, ${ }^{b}$ Changyun Miao, ${ }^{a}$ and \\ Chunxiao Tang ${ }^{\mathrm{a}}$ \\ ${ }^{a}$ Tianjin Polytechnic University, School of Electronics and Information Engineering, No. 399 Binshuixi Road, Xiqing District, Tianjin 300387, China \\ bUniversity of Wollongong, School of Physics, Faculty of Engineering, Wollongong, New South Wales 2522, Australia
}

\begin{abstract}
A highly efficient polarization-independent output grating coupler was optimized and designed based on silicon-on-insulator used for silica-based hybrid photodetector integration in an arrayed waveguide grating demodulation-integrated microsystem. The finite-difference time-domain (FDTD) method optimizes coupling efficiency by enabling the design of the grating period, duty cycle, etch depth, grating length, and polarizationdependent loss (PDL). The output coupling efficiencies of both the transverse electric (TE) and transverse magnetic (TM) modes are higher than $60 \%$ at 1517 to $1605 \mathrm{~nm}$ and $\sim 67 \%$ at around $1550 \mathrm{~nm}$. The designed grating exhibits the desired property at the 3-dB bandwidth of $200 \mathrm{~nm}$ from 1450 to $1650 \mathrm{~nm}$ and a PDL $<0.5 \mathrm{~dB}$ of $110 \mathrm{~nm}$ from 1513 to $1623 \mathrm{~nm}$. The power absorption efficiency at $1550 \mathrm{~nm}$ for TE and TM modes reaches $78 \%$ and $70 \%$, respectively. Both the power absorption efficiency of TE mode and that of TM mode are over $70 \%$ in a broad band of 1491 to $1550 \mathrm{~nm}$. @ 2014 Society of Photo-Optical Instrumentation Engineers (SPIE) [DOI: 10.1117/1.OE.53.5.057105]
\end{abstract}

Keywords: silicon-on-insulator; grating coupler; polarization-independent; silica-based hybrid photodetector integration.

Paper 140306 received Feb. 25, 2014; revised manuscript received Apr. 8, 2014; accepted for publication Apr. 10, 2014; published online May 7, 2014.

\section{Introduction}

Silicon-on-insulator (SOI) offers important advantages, including high-refractive-index contrast, which allows a high integration scale and CMOS compatibility. ${ }^{1}$ Low power loss and high integration density in optical circuits have increased the importance of grating couplers with high coupling efficiency. Surface grating couplers ${ }^{2,3}$ have been widely used; however, they heavily depend on polarization. Thus, such couplers are usually designed to couple light in either transverse electric $(\mathrm{TE})^{4-6}$ or transverse magnetic (TM) ${ }^{7}$ polarizations. Researchers have produced SOI-based grating couplers with high coupling efficiency and great bandwidth. Zhou proposed a compact coupler with ultrabroad bandwidth and high coupling efficiency. The TE-mode coupling efficiencies of this coupler can be obtained at the 3-dB bandwidth of $160 \mathrm{~nm}$ from 1390 to $1550 \mathrm{~nm}$, whereas the coupling efficiency of $\sim 50 \%$ can be achieved at around $1550 \mathrm{~nm} .{ }^{8} \mathrm{Li}$ designed a grating coupler on an SOI wafer operating at $1550 \mathrm{~nm}$ based on ultrathin 50 -nm silicon geometry. The insertion loss of these couplers was $-3.7 \mathrm{~dB}$, and the back-reflection loss was $-14 \mathrm{~dB} .{ }^{9}$ Alonso-Ramos et al. ${ }^{10}$ designed an SOI-based dual-polarization grating coupler with a coupling efficiency of $-2.8 \mathrm{~dB}$.

Our previous works proposed and studied the novel integration of arrayed waveguide grating (AWG) demodulation systems shown in Fig. 1, which is a new kind of optical fiber grating demodulation scheme that is suitable for optoelectronic integration. The system included an on-chip light source, an opti-waveguide, an multimode interface (MMI) coupler, ${ }^{11}$ an fiber Bragg grating (FBG) array, an AWG, ${ }^{12}$ InP/InGaAs photodetector array, and a signal-processing unit. The integrated microsystem has the advantages of

*Address all correspondence to: Hongqiang Li, E-mail: lihongqiang@tjpu.edu .cn compact form, high integration, low cost, stability, and easy implementation. The current work aims to study the grating coupler among the AWG output waveguide to photodetector, which was used in silica-based hybrid integrated photodetectors to diffract light from the SOI nanophotonic waveguide into the photodetector and with a spin-coated benzocyclobutene (BCB) layer in the middle. A schematic cross section is shown in Fig. 1(b).

In this article, we propose a highly efficient grating coupler designed for silica-based hybrid photodetector integration in an AWG demodulation-integrated microsystem. This coupler efficiently couples light from both TE and TM modes between SOI nanophotonic waveguides and photodetectors. The couplers are simulated and optimized by the FDTD method. Compared with other grating couplers, the output grating coupler designed in this study has a large size $\left(\right.$ area $=25 \times 25 \mu \mathrm{m}^{2}$ ) and exhibits high efficiency and great bandwidth. Power absorption efficiencies for TE and TM modes reach $78 \%$ and $70 \%$, respectively, at $1550 \mathrm{~nm}$. The minimum feature size is also larger than $200 \mathrm{~nm}$, thus enabling the use of i-line lithography and steppers in pattern definition.

\section{Theory and Structure}

A schematic diagram of the proposed SOI-based grating coupler structure is shown in Fig. 2. The grating structure is designed in a 220-nm-thick silicon waveguide layer on a 2- $\mu$ m-thick buried oxide. A cladding layer, such as $\mathrm{SiO}_{2}$ cladding and air cladding, is often used to protect the silicon waveguide layer. However, several applications, such as silicon waveguides with heterogeneous integration of photoelectric detectors, require BCB cladding [Fig. 2(b)]. To achieve high performance, some design parameters, such

0091-3286/2014/\$25.00 (C) 2014 SPIE 


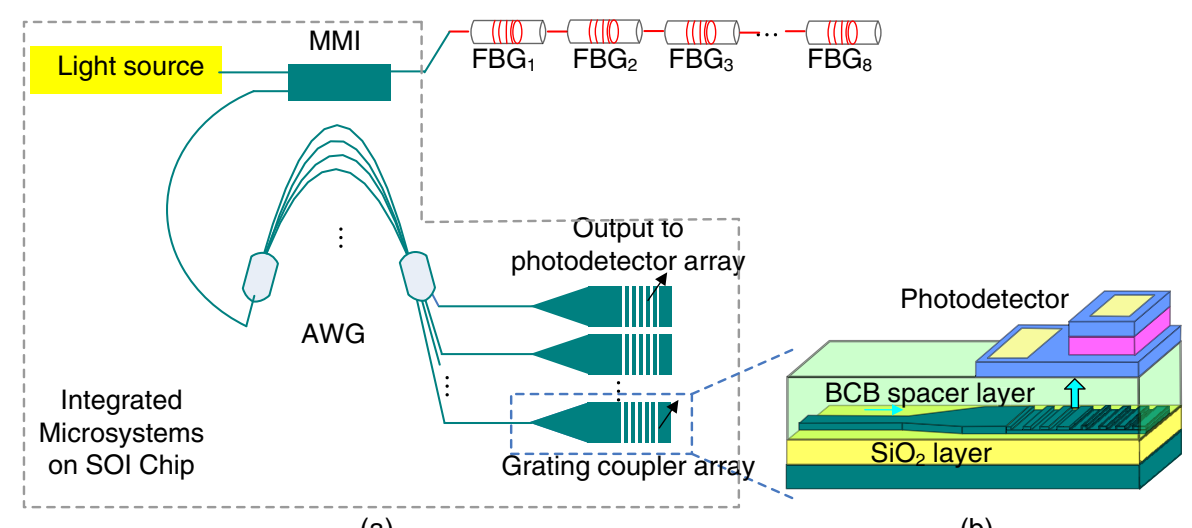

(a)

(b)

Fig. 1 (a) Arrayed waveguide grating demodulation-integrated microsystem based on silicon-on-insulator (SOI). (b) Coupling scheme for III-V photodetectors bonded to SOI nanophotonic waveguides.

as grating period $\Lambda$, duty cycle $\mathrm{DC}(\mathrm{DC}=W / \Lambda)$, etch depth ed, grating length $L, \mathrm{BCB}$ bonding layer thickness $t_{\text {clad }}$ are considered. As shown in Fig. 2, incident power $P_{\text {in }}$ is divided into four parts throughout the grating: $P_{\text {up }}, P_{\text {down }}, P_{\mathrm{R}}$, and $P_{\mathrm{T}}$. Coupling efficiency $\eta$ is defined as $\eta=P_{\text {up }} / P_{\text {in }}$.

The diffraction equation for output coupling (Fig. 2) is described as

$n_{\mathrm{eff}} \cdot \Lambda-n_{c} \cdot \Lambda \cdot \sin \theta=m \cdot \lambda$,

where $n_{\text {eff }}$ denotes the effective index of grating, $\Lambda$ denotes the grating period, $n_{c}$ denotes the effective index of the top cladding layer, $\theta$ denotes the output coupling angle, $m$ is an integer denoting the diffraction order, and $\lambda$ denotes the wavelength of the out-coupled wave. The diffraction order normally used for coupling is the first order (i.e., $m=1$ ). Thus, the Bragg condition for a grating coupler can be simplified to

$\Lambda=\frac{\lambda}{n_{\mathrm{eff}}-n_{c} \cdot \sin \theta}$.

The effective index of grating can be calculated as follows: $2.85\left(n_{\text {eff } 1}\right)$ for the $220-n m-S i$ layer and $2.34\left(n_{\text {eff } 2}\right)$ for the shallow-etched Si layer. Then, the effective index of grating can be calculated according to $n_{\mathrm{eff}}=n_{\mathrm{eff} 1} / 2+$ $n_{\text {eff } 2} / 2$ (i.e., $2.595 ; \lambda=1550 \mathrm{~nm} ; n_{c}=1.45$ ). Based on this

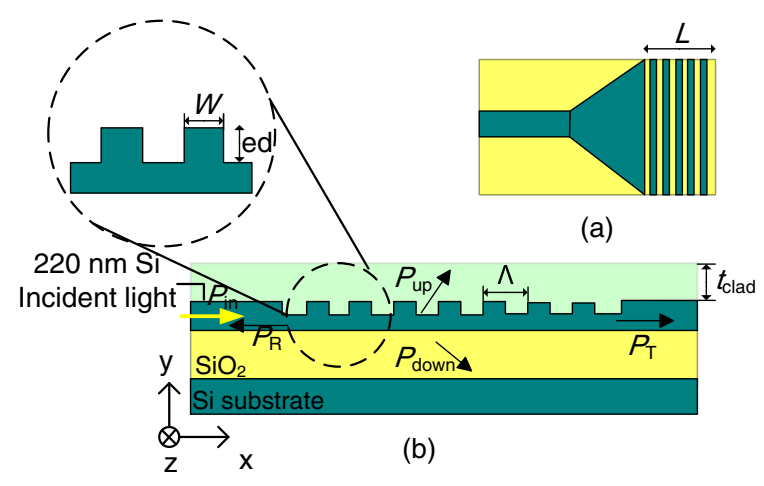

Fig. 2 Schematic diagram of the grating coupler: (a) Top view and (b) side view. original value, $\Lambda$ is optimized to meet practical requirements. As for the structure, light injected from the waveguide goes through the grating. Some light is reflected back to the waveguide, some other light goes through the grating into the subsequent waveguide, and yet some other light is diffracted by the grating upward to the cladding layer or down to the buffer layer and even the Si substrate. Light in the waveguide grating dissipates.

Conventional Si-based grating couplers commonly exhibit polarization dependence. The designed grating device usually couples only the input or output of either the TE or TM mode. This feature is determined by the grating coupler's Bragg condition, under which TE and TM modes with the same waveguide thickness have different effective refractive indices.

The polarization characteristics of the device are described by polarization-dependent loss (PDL):

$\mathrm{PDL}=10 \times \log \left|\frac{\eta_{\mathrm{TE}}}{\eta_{\mathrm{TM}}}\right|$,

where $\eta_{\mathrm{TE}}$ is the efficiency of the TE mode (for the coupler, the coupling efficiency) and $\eta_{\mathrm{TM}}$ is the efficiency of the TM mode. The size of PDL reflects the polarization correlation of the device. The smaller the PDL, the higher the polarization independence of the device.

For the high detector efficiency consideration of the system of silica-based hybrid photodetector integration, the thickness of the $\mathrm{SiO}_{2}$ buffer and $\mathrm{BCB}$ bonding layers as a cavity is formed by the reflection at the $\mathrm{BCB} / \mathrm{InP}$ and $\mathrm{SiO}_{2} / \mathrm{Si}$ substrate interfaces. The thickness of silicon dioxide of the SOI should obey the reflection condition as follows: ${ }^{13}$

$d_{\mathrm{SiO}_{2}} \approx(2 b+1) \frac{\lambda}{4 n_{\mathrm{SiO}_{2}}}$,

where $n_{\mathrm{SiO} 2}$ is the refractive index of $\mathrm{SiO}_{2}\left(n_{\mathrm{SiO} 2}=1.45\right)$, and $b$ is an integer. The thickness of the BCB layer should act as an antireflection film to help light pass into the detector, as follows: ${ }^{13}$ 


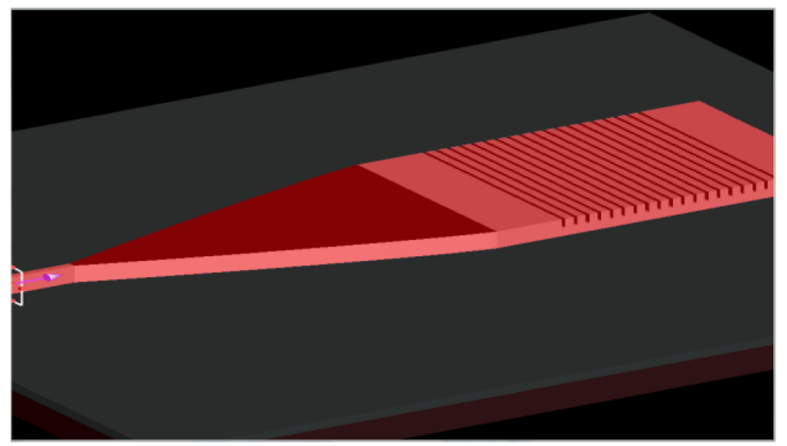

Fig. 3 Grating coupling structure model for three-dimensional FDTD simulation.

$$
d_{\mathrm{BCB}} \approx \frac{c \lambda}{2 n_{\mathrm{BCB}}} .
$$

\section{Design and Optimization}

The FDTD method is used to model the grating couplers and simulate their performance as a function of geometrical parameters. This method is selected because it easily manages complex electromagnetic structures, and because the gratings are significantly perturbed. We model the waveguide grating coupler with boundaries having perfectly matched layers. Designing a grating coupler follows a certain procedure. The first step is to achieve the initial conditions of the desired grating coupler through theoretical calculations, such as those mentioned above. The second step is to optimize the performance of the initial design in terms of various parameters, such as grating period, duty cycle, and etch depth. The grating PDL must also be examined. The total coupling efficiency obtained by three-dimensional (3-D) FDTD simulation, and a 3-D model of the grating coupler is shown in Fig. 3.

Equation (2) shows that the central wavelength of the grating coupler is proportional to the grating period. Figure 4 shows the simulation results for different grating periods. The wavelength is kept at 1450 to $1650 \mathrm{~nm}$ for TE mode, $\mathrm{DC}=0.6$, ed $=0.1 \mu \mathrm{m}$. Changing the grating period from 640 to $760 \mathrm{~nm}$ shifts the central wavelength of the

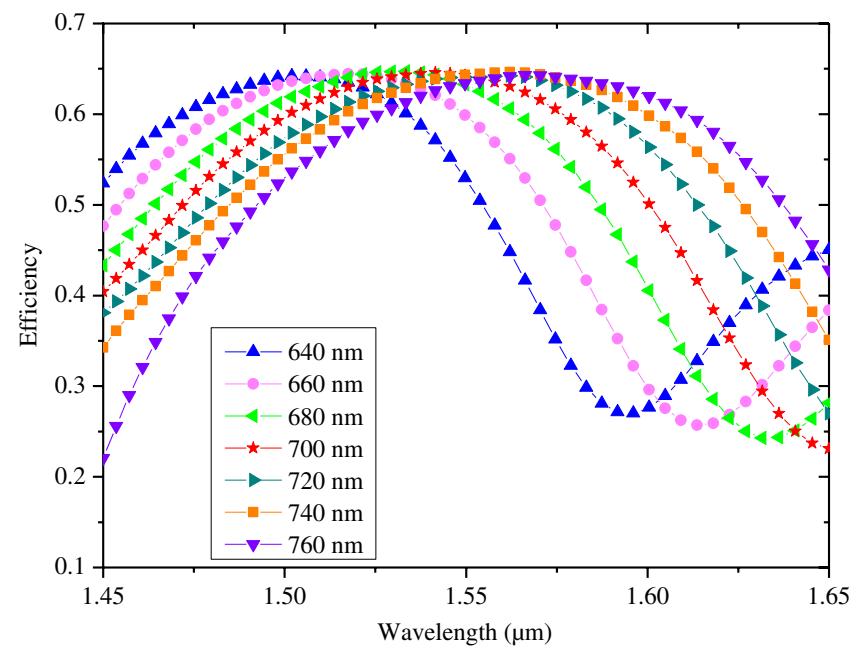

Fig. 4 Variations of period. grating coupler from 1500 to $1650 \mathrm{~nm}$. The red shifts in the central wavelength are consistent with our analytical calculation. Under these conditions, the period is further optimized at $1550 \mathrm{~nm}$. Thus, the maximum efficiency $(63 \%)$ is reached at $707 \mathrm{~nm}$.

Figure 5 shows the simulation results for different duty cycles. Grating period is kept constant at $707 \mathrm{~nm}$, etch depth is kept constant at $0.1 \mu \mathrm{m}$, and the duty cycle is varied from 0.4 to 1.0. The central wavelength shifts from 1500 to $1570 \mathrm{~nm}$ with varying duty cycles. At the wavelength of $1550 \mathrm{~nm}$, the maximum coupling efficiency is reached at $\mathrm{DC}=0.8$. The groove width is smaller than $200 \mathrm{~nm}$. However, the minimum groove width for the manufacturer (IME, Singapore) is $200 \mathrm{~nm}$. Thus, we use 0.7 as the duty cycle.

After the period of the output grating coupler and the duty cycle of the grating is optimized, the grating period selected is $707 \mathrm{~nm}$, and the grating groove width is $212 \mathrm{~nm}$. Increasing the etch depth decreases the effective index of refraction of the shallow-etched area and thus reduces $n_{\text {eff }}$. The effective index of refraction is proportional to the central grating wavelength; therefore, the etch depth of the grating coupler is inversely proportional to the central grating wavelength. Figure 6(a) shows the relationship between the efficiency of the grating coupler and the gratings etch depth. The grating period and duty cycle are kept constant, and the etch depth of the grating coupler is varied from 50 to $100 \mathrm{~nm}$. The central wavelength shows an orange shift with increasing etch depth, consistent with our analytical calculation. Changes in the etch depth from 0 to $220 \mathrm{~nm}$ at the wavelength of $1550 \mathrm{~nm}$ are then analyzed. Changing the etch depth from 0 to $50 \mathrm{~nm}$ significantly changes the coupling efficiency [Fig. 6(b)]. The coupling efficiency exceeds $60 \%$ and only slightly changes when the etch depth changes from 50 to $220 \mathrm{~nm}$. Figures 6(a) and 6(b) suggest that etching tolerance is larger at the wavelength of $1550 \mathrm{~nm}$. In addition, following the technical dimension requirements of IME, we choose $70 \mathrm{~nm}$ as the etch depth.

To improve the integration density in optical circuits, the feature sizes of grating coupler must be shrunk step by step. Overcoupling or undercoupling occurs when the grating is too long or too short, which reduces coupling efficiency accordingly. Thus, an optimum grating length must be chosen. The relationships between the grating length and

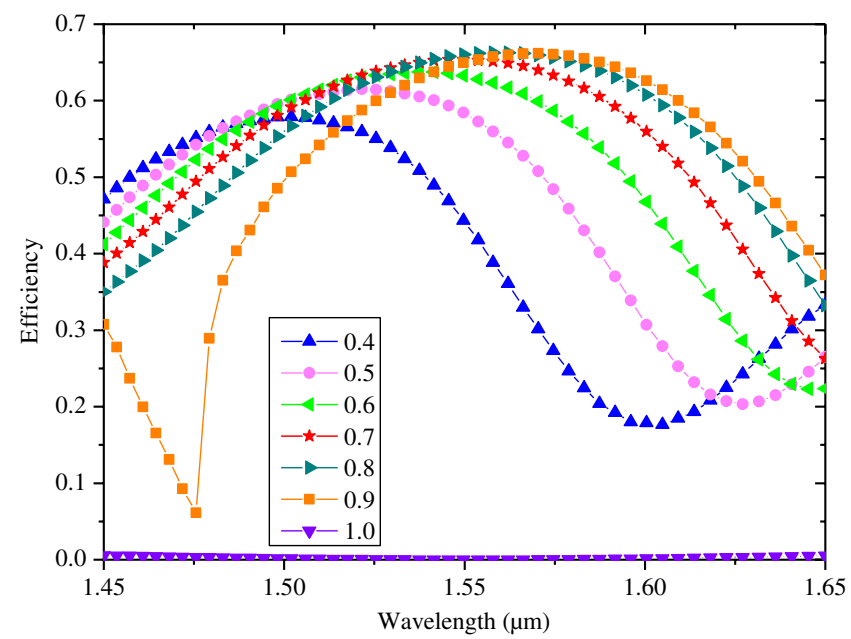

Fig. 5 Variations of duty cycle. 


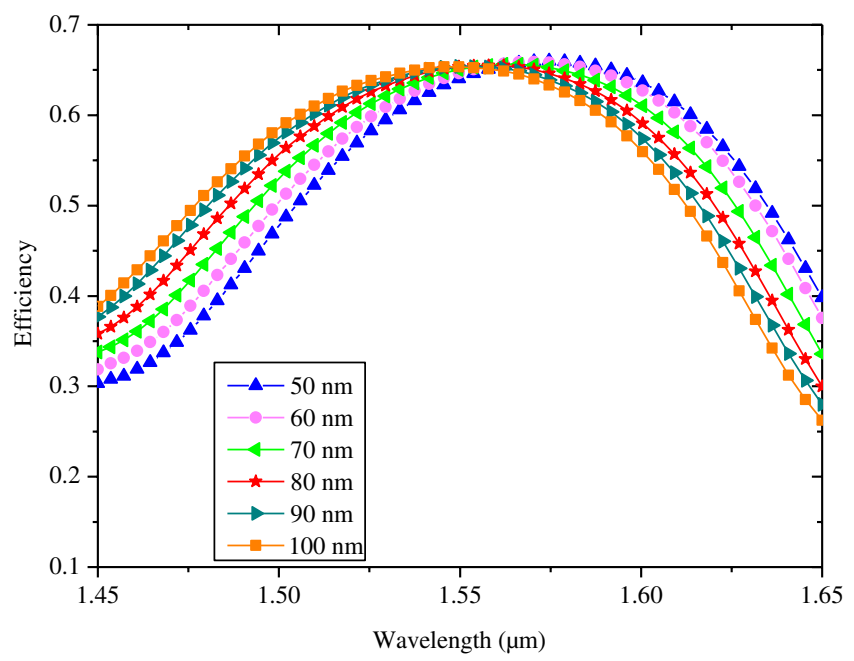

(a)

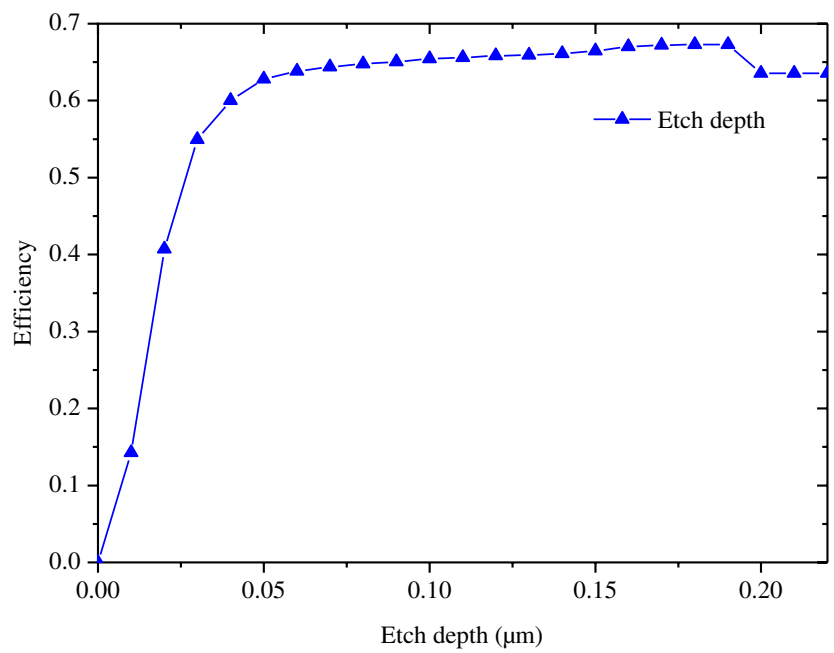

(b)

Fig. 6 (a) Variations of etch depth. (b) Relationship between coupling efficiency and etch depth.

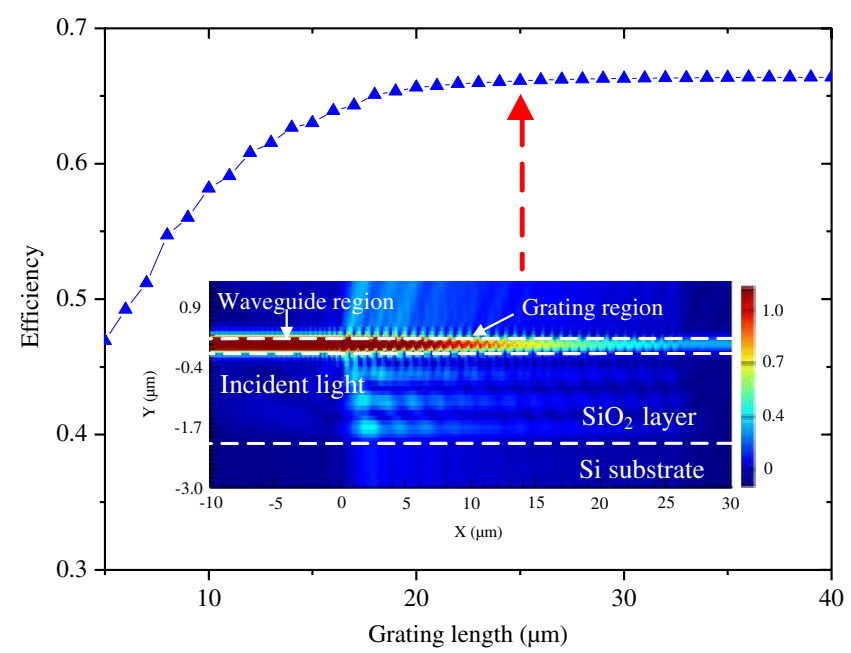

Fig. 7 Relationships between coupling efficiency and grating length. Inset: Optical field of the output grating. (The grating length is $25 \mu \mathrm{m}$ and coupling efficiency $\sim 67 \%$ at around $1550 \mathrm{~nm}$ ).

efficiency are shown in Fig. 7. The photosensitive area of the photoelectric detector used in this study is a circle with a diameter of $55 \mu \mathrm{m}$. Thus, the grating length will be fixed at $25 \mu \mathrm{m}$ in the simulation. The optical field of the output grating calculated by the FDTD method is shown in the inset of Fig. 7. All important parameters used in the simulation are given in Table 1.

The relationships between the coupling efficiency and wavelength of the TE and TM modes are shown in Fig. 8(a). These figures also show the optical field distribution of the TE and TM modes coupling at $1550 \mathrm{~nm}$. The output coupling efficiencies of the TE and TM modes exceed 60\% at 1517 to $1605 \mathrm{~nm}$ (a high 69\% for
TM mode at $1470 \mathrm{~nm}$ ). High coupling efficiency for both modes and high polarization independence are achieved at 1513 to $1622 \mathrm{~nm}$. The PDL of the grating is shown in Fig. 8(b). The grating exhibits high polarization independence around $1550 \mathrm{~nm}$. The PDL of the device is within $0.5 \mathrm{~dB}$ at 1513 to $1623 \mathrm{~nm}$. High coupling efficiency for both TE and TM modes and high polarization independence are achieved in a wide wavelength range of 1513 to $1623 \mathrm{~nm}$. Table 2 shows a comparison of grating couplers designed in recent years. The output light directions in the TE and TM modes differ. In the TE mode, a 73-deg angle exists between the direction of the coupling output light and the wave vector in the direction of the input light at $1550 \mathrm{~nm}$. In the TM mode, a 114-deg angle exists between the directions of the coupling output light and input light at the same wavelength. This characteristic requires improvement. However, if the structure is used in integrated optical detectors, these problems will not significantly influence the short distance between the light-receiving surface and the grating output surface. Therefore, we use this structure in silica-based hybrid integrated photodetectors.

An InP/InGaAs photodetector is bonded onto this grating coupler with a spin-coated BCB layer in the middle to achieve silica-based hybrid integrated photodetectors. $\mathrm{SiO}_{2}$ buffer layer and BCB layer have reflection and antireflection effects on power absorption efficiency, so we choose the thickness of the $\mathrm{SiO}_{2}$ buffer layer and BCB layer as $2 \mu \mathrm{m}$ and $440 \mathrm{~nm}$ by optimization. The relationships between the wavelength and the power absorption efficiency for TE and TM modes are shown in Fig. 9. A power absorption efficiency of $78 \%$ for TE mode and that of $70 \%$ for TM mode are achieved at $1550 \mathrm{~nm}$. Power absorption efficiencies higher than $70 \%$ for both TE and TM modes are realized in a wide wavelength range of 1491 to $1550 \mathrm{~nm}$.

Table 1 Design parameters of grating coupler.

\begin{tabular}{lccccc} 
Si layer thickness & $\mathrm{SiO}_{2}$ layer thickness & Period & Duty cycle & Etching depth & Grating length \\
\hline$d_{\mathrm{Si}}=0.22 \mu \mathrm{m}$ & $d_{\mathrm{SiO} 2}=2 \mu \mathrm{m}$ & $\Lambda=0.707 \mu \mathrm{m}$ & $\mathrm{DC}=0.7$ & ed $=0.07 \mu \mathrm{m}$ & $L=25 \mu \mathrm{m}$ \\
\hline
\end{tabular}




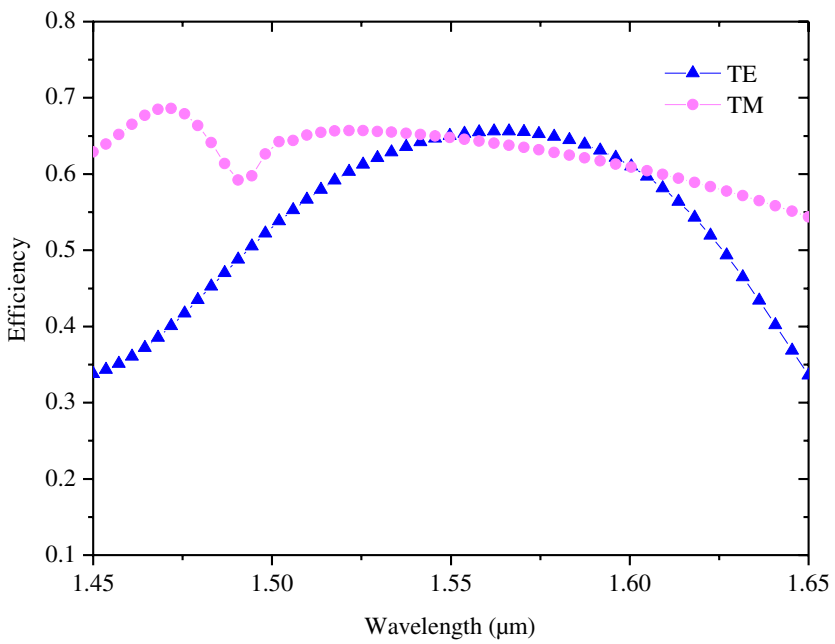

(a)

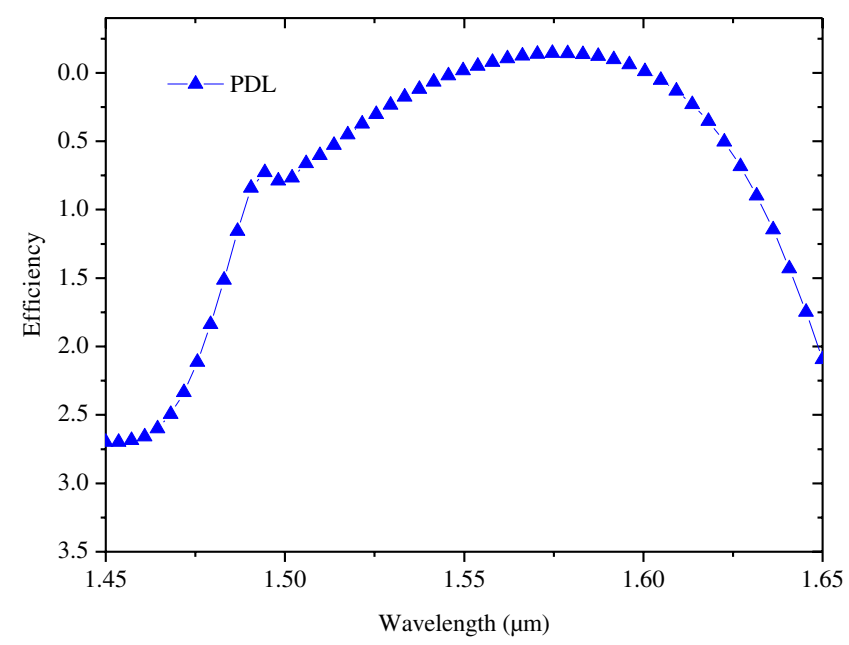

(b)

Fig. 8 (a) Relationships between the coupling efficiency and the wavelength for both the transverse electric (TE) and transverse magnetic (TM) modes. (b) The polarization-dependent loss of the grating.

Table 2 Comparison of grating couplers.

\begin{tabular}{lcccc} 
Time & Author & Grating coupler types and materials & Coupling efficiency $(\%)$ & Wavelength $(\mu \mathrm{m})$ \\
\hline 2009 & Schmid et al. $^{14}$ & Fully etched grating coupler (SOI) & 49 & 1.55 \\
2010 & Zhu et al. $^{15}$ & Grating coupler (SOI) & 46 & 1.55 \\
2010 & Halir et al. $^{7}$ & Continuously apodized grating couple (SOI) & 43 & 1.53 \\
2011 & Zhou et al. $^{8}$ & Multilevel grating coupler (SOI) & 67.5 & 1.46 \\
2013 & Zhang et al. $^{16}$ & Vertical grating coupler (SOI) & 37 & 1.55 \\
2014 & Present paper & Grating coupler (SOI) & 67 & 1.55 \\
\hline
\end{tabular}

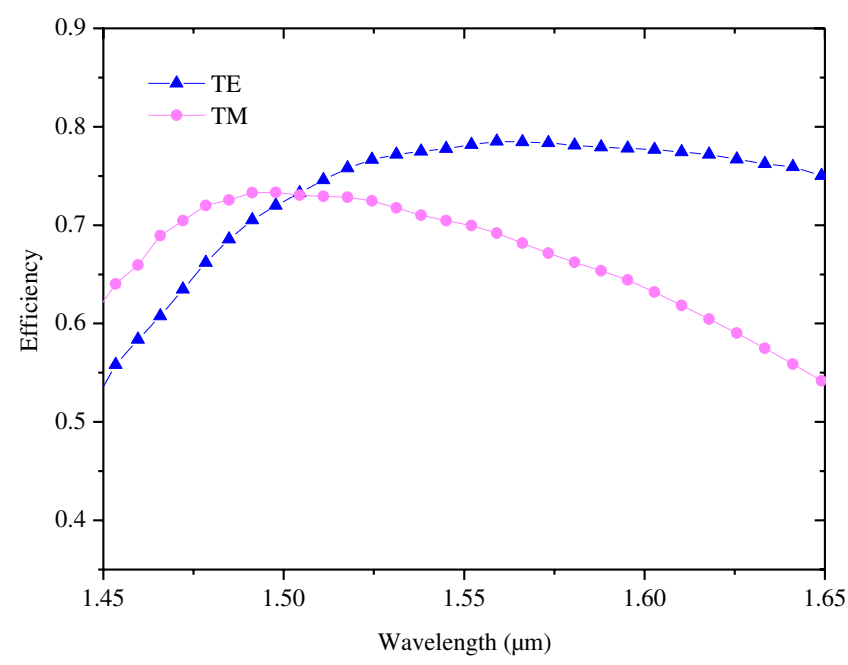

Fig. 9 Relationships between the power absorption efficiency and the wavelength for both the TE and TM modes.

\section{Conclusion}

In conclusion, we propose a highly efficient polarizationindependent grating coupler that couples SOI nanophotonic waveguides with photodetectors for silica-based hybrid integrated photodetectors. The coupling efficiencies of the TE and TM modes exceed $60 \%$ at 1517 to $1605 \mathrm{~nm}$. The coupler also has a $3-\mathrm{dB}$ bandwidth of $200 \mathrm{~nm}$ at 1450 to $1650 \mathrm{~nm}$ and a coupling efficiency of $\sim 67 \%$ at $1550 \mathrm{~nm}$, comparable with the efficiency of shallow-etched gratings. High power absorption efficiency of $\mathrm{InP} / \mathrm{InGaAs}$ photodetector for both TE and TM modes is acquired in a broad band. Also, the power absorption efficiency at $1550 \mathrm{~nm}$ for TE and TM modes reaches $78 \%$ and $70 \%$, respectively. Both the power absorption efficiency of TE mode and that of TM mode are over $70 \%$ in a broad band of 1491 to $1550 \mathrm{~nm}$. The simulation results and theoretical analysis show that the grating coupler is useful for integrated optical circuits. The coupler can be fabricated by conventional and mature CMOS technology. Large and technically enhanced grating couplers for silica-based hybrid integrated photodetectors can be developed to improve the coupling efficiency and bandwidth of the grating coupler.

\section{Acknowledgments}

This work is supported by the National Natural Science Foundation of China (Nos. 61177078, 61307094, 31271871), the Specialized Research Fund for the Doctoral Program of Higher Education of China (No. 20101201120001), and Tianjin Research Program of 
Application Foundation and Advanced Technology (No. 13JCYBJC16800).

\section{References}

1. B. Jalali and S. J. Fathpour, "Silicon photonics," J. Lightwave Technol. 24(12), 4600-4615 (2006)

2. C. Chen and P. Berini, "Grating couplers for broadside input and output coupling of long-range surface plasmons," Opt. Express 18(8), 8006-8018 (2010)

3. D. Taillaert et al., "An out-of-plane grating coupler for efficient butt-coupling between compact planar waveguides and single-mode fibers," IEEE J. Quantum Electron. 38(7), 949-955 (2002).

4. H. Y. Chen and K. C. Yang, "Design of a high-efficiency grating coupler based on a silicon nitride overlay for silicon-on-insulator waveguides," Appl. Opt. 49(33), 6455-6462 (2010).

5. D. Taillaert, P. Bienstman, and R. Baets, "Compact efficient broadband grating coupler for silicon-on-insulator waveguides," Opt. Lett. 29(23), 2749-2751 (2004).

6. G. L. Li et al., "Ultralow-loss, high-density SOI optical waveguide routing for macrochip interconnects," Opt. Express 20(11), 12035-12039 (2012).

7. R. Halir et al., "Continuously apodized fiber-to-chip surface grating coupler with refractive index engineered subwavelength structure," Opt. Lett. 35(19), 3243-3245 (2010).

8. W. Zhou et al., "Ultrabroad bandwidth and high-coupling-efficiency compact coupler using multilevel grating structure," Proc. SPIE 8191, 819120 (2011).

9. L. He et al., "Ultrathin silicon-on-insulator grating couplers," IEEE Photonics Technol. Lett. 24(24), 2247-2249 (2012).

10. C. Alonso-Ramos et al., "Polarization-independent grating coupler for micrometric silicon rib waveguides," Opt. Lett. 37(17), 3663-3665 (2012).

11. H. Li et al., "Experimental demonstration and analysis of compact silicon-nanowire-based couplers," Opt. Eng. 52(6), 064003 (2013).

12. H. Li et al., "Practical fabrication and analysis of an optimized compact 8-channel silicon arrayed waveguide grating," Opt. Eng. 52(6), 064602 (2013).

13. J. Mu, H. Zhang, and W. Huang, "Design of waveguide bragg gratings with strong index corrugations," J. Lightwave Technol. 26(12), 1596-1601 (2008)
14. B. Schmid, A. Petrov, and M. Eich, "Optimized grating coupler with fully etched slots," Opt. Express 17(13), 11066-11076 (2009).

15. Y. Zhu et al., "High efficiency and broadband width grating coupler between nanophotonic waveguide and fibre," Chin. Phys. B. 19(1), 014219 (2010).

16. Z. Zhang et al., "CMOS-compatible vertical grating coupler with quasi Mach-Zehnder characteristics," IEEE Photonics Technol. Lett. 25(3), 224-227 (2013).

Hongqiang $\mathrm{Li}$ received his $\mathrm{BE}, \mathrm{MSc}$, and $\mathrm{PhD}$ degrees from Tianjin Polytechnic University. $\mathrm{He}$ is a professor in the School of Electronics and Information Engineering, Tianjin Polytechnic University. His research interests lie in the area of optical fiber grating sensor demodulation and optoelectronics integration.

Yu Liu is working on her master's degree in the School of Electronics and Information Engineering, Tianjin Polytechnic University.

Meiling Zhang is a vice professor in the School of Textile, Tianjin Polytechnic University.

Wenqian Zhou is working on his master's degree in the School of Electronics and Information Engineering, Tianjin Polytechnic University.

Cheng Zhang is a lecturer in the School of Electronics and Information Engineering, Tianjin Polytechnic University.

Enbang Li is a professor in the School of Physics, Faculty of Engineering, University of Wollongon.

Changyun Miao is a professor in the School of Electronics and Information Engineering, Tianjin Polytechnic University.

Chuanxiao Tang is a lecturer in the School of Electronics and Information Engineering, Tianjin Polytechnic University. 